\title{
Informed Sampling for Diversity in Concept-to-Text NLG
}

\author{
Giulio Zhou \\ Huawei Noah's Ark Lab \\ London, UK \\ giuliozhou@huawei.com
}

\author{
Gerasimos Lampouras \\ Huawei Noah's Ark Lab \\ London, UK \\ gerasimos. lampouras@huawei.com
}

\begin{abstract}
Deep-learning models for language generation tasks tend to produce repetitive output. Various methods have been proposed to encourage lexical diversity during decoding, but this often comes at a cost to the perceived fluency and adequacy of the output. In this work, we propose to ameliorate this cost by using an Imitation Learning approach to explore the level of diversity that a language generation model can reliably produce. Specifically, we augment the decoding process with a metaclassifier trained to distinguish which words at any given timestep will lead to high-quality output. We focus our experiments on conceptto-text generation where models are sensitive to the inclusion of irrelevant words due to the strict relation between input and output. Our analysis shows that previous methods for diversity underperform in this setting, while human evaluation suggests that our proposed method achieves a high level of diversity with minimal effect to the output's fluency and adequacy.
\end{abstract}

\section{Introduction}

The use of deep-learning models for language generation tasks has become prevalent in recent years as they achieve high performance without manually engineered rules or features (Wen et al., 2015b; Mei et al., 2016; Dušek et al., 2018). However, while the produced texts are qualitatively acceptable according to most evaluation criteria, they are often repetitive or disfluent when multiple diverse outputs are needed. This problem is attributed to using the maximum-likelihood objective function for training as it encourages the generation of highly frequent words and sentence structures, i.e. models overfit and do not learn to exploit the lexical and structural diversity that is present in the dataset $(\mathrm{Li}$ et al., 2016).

Here we focus on concept-to-text Natural Language Generation (NLG), where the input is a meaning representation (MR) and the output is an ut- terance expressing the input in natural language. Due to the stricter relation between input and output, it is more challenging to promote diversity in concept-to-text than other language generation tasks. Diverging from greedy inference can lead to error propagation that negatively affects the output's relevance to the input. However, assuming the output is sequentially decoded, most research on concept-to-text diversity focuses on sampling over the probability distribution (Wen et al., 2015b).

More complex decoding strategies have been proposed for the related task of open-domain NLG, where the input is a natural language context and the output is a relevant response. Fan et al. (2018) limit the decoding distribution to a fixed number of the Top- $k$ words (Top- $k$ Sampling), while Holtzman et al. (2020) limit the distribution to the largest subset of words whose cumulative probability does not exceed a predefined parameter $p$ (Nucleus Sampling). Nucleus Sampling improves over Top- $k$ by retaining a dynamic number of words per decoding step, but the probability mass $p$ remains a constant parameter. However, these strategies are sensitive to their parameters $k$ and $p$ and there is no established methodology to tune them so that the output fluency and adequacy do not suffer while also achieving high diversity.

In this paper, we propose Informed Sampling for diversity, i.e. to sample amongst reliable words that lead to diverse output but are not liable to lead to disfluent word sequences through error propagation. To distinguish which words in the decoding distribution can be reliably sampled by the NLG model, we employ a meta-classifier that leverages a diversity-specific training signal. Our approach is only applied during decoding and is orthogonal to the architecture of the NLG model, which we assume as pretrained. Unlike previous decoding strategies, Informed Sampling does not depend on manually tuned parameters.

As there is no explicitly annotated data for In- 


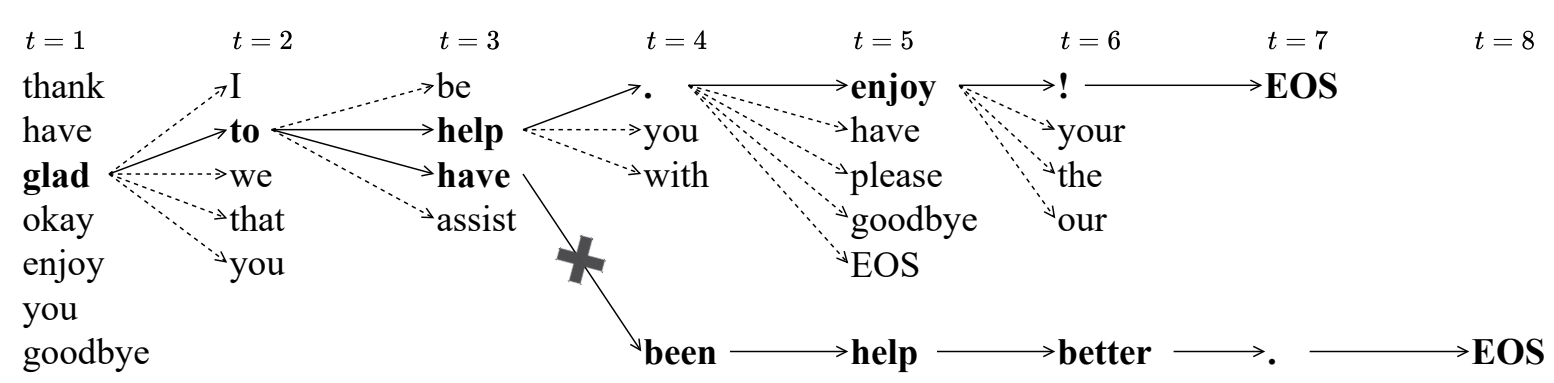

Figure 1: Example decoding for [INFORM(WELCOME); INFORM(BYE)]; diverging at the third time step.

formed Sampling, we adapt three Imitation Learning (IL) frameworks to train the meta-classifier; IL is a family of meta-learning frameworks that train models based on expert demonstrations. We design an expert policy as to infer which words are reliable based on what the NLG model can produce without negatively affecting the output's quality. Through this, the meta-classifier is fitted to the level of diversity captured by the NLG model.

We present experimental analysis on the application of IL to the meta-classifier and compare against related approaches. Additionally, this paper explores the concept-to-text application of diversity methods originally proposed for open-domain NLG. Automatic and human evaluation suggests that Informed Sampling produces diverse output while maintaining its fluency and adequacy.

\section{Related Work}

There have been a number of different approaches to encourage output diversity in open-domain NLG. $\mathrm{Li}$ et al. (2016) propose mutual information maximization as a diversity focused objective function, while Zhang et al. (2018) propose variational information maximization in combination with adversarial learning. Zhao et al. (2017) produce diverse output by augmenting the input encoding with diversity-specific information through Conditional Variational Autoencoders. Going further with modifying the encoding, Gao et al. (2019) reshape the whole embedding space of the input, arguing that a more structured latent space leads to more diverse output. We explore the application of these methods to concept-to-text NLG in later sections, but we find that they underperform compared to their open-domain use. These methods promote semantic diversity, and might be incompatible with concept-to-text where the output semantics are strictly bounded by the input.

Research on neural output diversity for concept- to-text NLG is limited and mostly focused on different decoding strategies (e.g. beam search). Most recently, Deriu and Cieliebak (2017) proposed "forcing" the output of the first decoding step, arguing that greedy inference from different starting points leads to diverse but fluent sentences. They achieve this by augmenting the input to bias the first step of the decoding process towards particular words observed in the data. However, the application of their method is limited to the first decoding step.

Imitation Learning frameworks have been applied on a variety of structured prediction NLP tasks, such as dependency (Goldberg and Nivre, 2013) and semantic parsing (Vlachos and Clark, 2014). Most related to this work, the LOLS framework was applied to concept-to-text NLG from unaligned data (Lampouras and Vlachos, 2016).

\section{Meta-Classifier for Diversity}

Concept-to-text NLG is the task of converting a machine-interpretable MR into natural language text. The input MR consists of one or more predicates; each predicate has a set of attributes and corresponding values. The predicate dictates the communication goal of the output text, while attributes and values dictate content. For example, the MR [INFORM(REST-NAME = MIZUSHI, OKASAN)] denotes that the output should inform the user of two restaurants called "Mizushi" and "Okasan". Concept-to-text datasets usually provide multiple output references per MR. Specifically, the MultiWOZ dataset (Budzianowski et al., 2018) provides 1872 distinct references for the MR [INFORM(WELCOME); INFORM(BYE)], e.g. "Glad to help. Enjoy!", "Glad to assist you. Goodbye."

We treat NLG as a structured prediction problem, where the output is a sequence of words constructed via sequential decoding. Informed Sampling is orthogonal to the architecture of the NLG model, only assuming a sequential decoding process. Figure 1 
shows a partial example of the diversity exhibited by a trained NLG model, for the previously mentioned MR. At each timestep we can examine the distribution that results from decoding and sample accordingly to promote diversity; the words are shown in descending probability. However, only a subset of words in the vocabulary will lead to fluent and adequate sequences. As mentioned before, we denote these as reliable words. For example, in $t=3$ choosing the word "have" seems like a sensible choice given the history; one can imagine that this may lead to an output like "glad to have been of help!" Unfortunately, due to the NLG model being imperfect, this will actually lead to the disfluent output "glad to have been help better." On the contrary, the word "assist" has less probability than "have" but it leads to the same subtree as "help", and to fluent output.

As briefly mentioned in the introduction, we propose to use a meta-classifier (see Figure 2), external to the NLG model, that learns to distinguish which words in the decoding distribution are reliable. The meta-classifier is a simple feed-forward neural network composed of alternating linear and ReLU layers ending with a softmax. It considers each word in the NLG model vocabulary individually, and the output for each is a probability distribution over values 0 and $1 ; 1$ denotes the word as reliable.

The input $\mathbf{c}$ for a given word is a concatenation of the NLG states and embeddings (eq. 1).

$$
\begin{aligned}
\mathbf{c}= & {\left[\mathbf{h}_{t}, W_{d c} \mathbf{d}_{t}, W_{w r} \mathbf{x}_{t+1}^{i},\right.} \\
& \left.W_{w r} \mathbf{x}_{t-2}, W_{w r} \mathbf{x}_{t-1}, W_{w r} \mathbf{x}_{t}\right]
\end{aligned}
$$

where $h_{t}$ is the hidden state at step $t, W_{d c}$ is the input representation weight matrix, $d_{t}$ represents input to be generated, $W_{w r}$ is a word embedding weight matrix, $x_{t}$ is the word at step $t$, and $x_{t+1}^{i}$ is the $i$-th word of the decoding distribution at $t+1$. In this paper we use notations specific to the SCLSTM architecture (Wen et al., 2015b). However, the input of the meta-classifier can be generalised as a concatenation of encoder, decoder hidden states and word embeddings.

From the meta-classifier's output we can infer a vocabulary-length binary vector $B$ that indicates which words are reliable. In order to also consider the NLG decoder's probability distribution, we only sample amongst the top consecutive reliable words in $B$ that are assigned a non-zero probability.

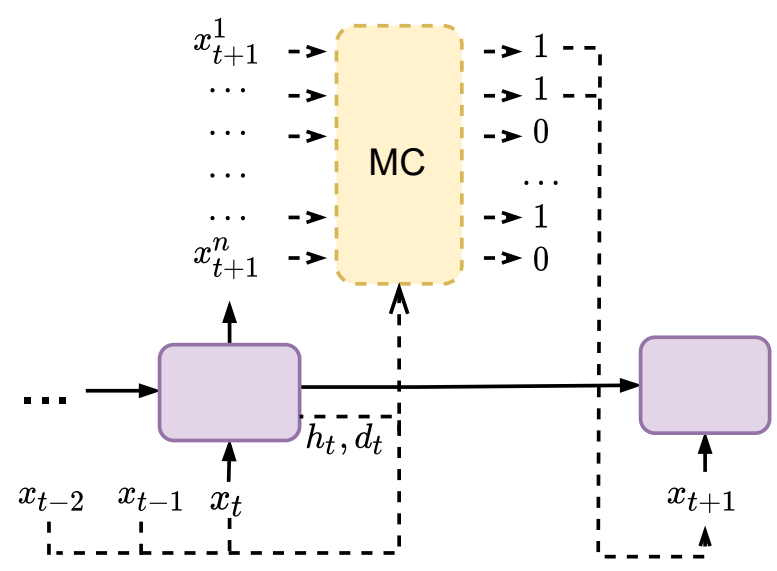

Figure 2: Overview of the meta-classifier (MC); dotted lines denote the MC, solid denotes NLG model.

\section{Imitation Learning}

Since diversity-specific labels are not explicitly available in the data, we employ an expert policy to infer which words are reliable, and use Imitation Learning (IL) approaches to mimic the expert. IL is a family of meta-learning frameworks, that train a policy $\pi$ using demonstrations provided by an expert $\pi^{r e f}$. In this work, the policy $\pi$ refers to the meta-classifier. The expert policy $\pi^{r e f}$ acts as a dynamic oracle that returns whether a word is reliable; we discuss the expert further in section 4.1.

We explore the application of three IL frameworks for training the meta-classifier: Exact Imitation, DAGGER (Ross et al., 2011) and Locally Optimal Learning to Search (Chang et al., 2015, LOLS). We will briefly explain how we adapt these frameworks, but a detailed explanation of the involved algorithms is out of the paper's scope.

Exact Imitation refers to training a policy $\pi$ directly on the labels provided by the expert policy $\pi^{r e f}$. In practice, for each training instance in our data, we use the underlying NLG model to generate a sentence. On each decoding step, we call $\pi^{r e f}$ to determine the reliable words and train $\pi$. We also sample the next word in the sentence using $\pi^{r e f}$. We note that the underlying NLG model remains constant throughout training and IL is applied solely on the meta-classifier.

DAGGER improves over Exact Imitation by generating the sentence using a mixture of the $\pi^{r e f}$ and $\pi$ policies, i.e. by sampling amongst the words considered reliable by either $\pi^{\text {ref }}$ or $\pi$. This way $\pi$ is exposed to sentences it would not have encountered solely using $\pi^{r e f}$ for sampling. In particular, it is exposed to sentences produced by $\pi$ itself, in a 


\begin{tabular}{l|l|l|l|l}
$x_{t}$ & $x_{t+1}^{i}$ & Greedy decoding & Prec $^{i}$ & Out \\
\hline my & favourite & is Itacho . do & 0.908 & 1 \\
my & personal & favorite is Itacho . & 1.0 & 1 \\
my & recommendation & is the hotel Hilton & 0.524 & 0 \\
my & computer & shows this . I & 0.658 & 0 \\
my & opinion & . I would recommend & 0.708 & 0 \\
my & suggestion & would be the Itacho & 1.0 & 1 \\
my & apologies & . I would suggest & 0.608 & 0
\end{tabular}

Table 1: Example of $\pi^{r e f}$ training signal inference.

sense exposing it to its own errors and thus helping ameliorate error propagation. As in Exact Imitation, we call $\pi^{r e f}$ to determine reliable words and train $\pi$. Before we apply DAGGER, we perform one iteration of Exact Imitation to initialise $\pi$.

LOLS generates the sentence using only the $\pi$ policy, again initialised via Exact Imitation. Additionally, at each decoding step the training signal is provided by either $\pi^{r e f}$ or $\pi$ according to a probability $p$. This probability is initially set to $p=1.0$, i.e. to always obtain the training signal via $\pi^{r e f}$, but it exponentially decays after every iteration with a rate of $p=(1-\beta)^{i}$, where $\beta$ is the learning rate. Further details on how $\pi$ can provide a training signal can be found in section 4.1.

DAGGER and LOLS iteratively adjust the training signal and increasingly expose $\pi$ to training instances that are more similar to what $\pi$ is likely to encounter during test time. This helps address error propagation, but also helps tune the meta-classifier to the level of diversity that the NLG model can comfortably produce. Specifically, LOLS has the advantage of potentially improving over $\pi^{r e f}$ as it exploits the training signal from $\pi$ itself.

\subsection{Inferring Training Signal from Policies}

During IL, we employ a dynamic oracle $\pi^{r e f}$ that determines whether a word $x_{t+1}^{i}$ is reliable. Due to the computational cost, $\pi^{r e f}$ is limited to consider only $i \in\{0 \ldots d\}$; in this work we consider the top $d=25$ words, which is the maximum number of consecutive reliable words as observed during preliminary training. This limit is not applied during decoding with the trained meta-classifier.

Intuitively, we need to examine whether the impact of each $x_{t+1}^{i}$ on the decoding process will lead to a fluent and adequate sentence. To obtain sentences that are affected by $x_{t+1}^{i}$, we force $x_{t+1}^{i}$ in step $t+1$ and use the NLG model to greedily generate the rest of the sentence. We then calculate the n-gram overlap between the $d$ sentences and a set of references. To make the calculations more consistent, we limit the produced sentences to the previous word $x_{t}, x_{t+1}^{i}$, and the next 4 words $x_{t+2}^{*} \ldots x_{t+5}^{*}$, similarly to the focused costing approach proposed by Goodman et al. (2016). If a sequence ends prematurely (e.g. by generating an $\langle e o s\rangle$ token), we pad it to the appropriate length.

An example application of $\pi^{r e f}$ is shown in Table 1 for the MR [INFORM(REST-NAME = ITACHO), REQUEST(REST-TYPE)]. Note that the previous word $x_{t}$ is the same for all examined $x_{t+1}^{i}$, while $x_{t+2}^{*} \ldots x_{t+5}^{*}$ differ. The $\mathrm{n}$-gram overlap is calculated via modified 4 -gram precision, i.e. BLEU-4 score (Papineni et al., 2002) without the brevity penalty. Since the expert hypotheses are all fixed in size, we cut the brevity penalty to speed up the calculation of the expert. The expert considers the words and corresponding modified 4-gram precisions $\operatorname{Prec}^{i}$ in ascending $i$, considering a word $i$ as reliable if $\operatorname{Prec}^{i} \geq \max \left(\operatorname{Prec}^{0}, \ldots, \operatorname{Prec}^{i-1}\right)$.

To promote more diversity through $\pi^{r e f}$, the aforementioned reference sets are obtained by decomposing the corresponding MR into its attributes, and then retrieving from the training instances all the references these attributes correspond to. For example, for [INFORM(WELCOME); INFORM(BYE)] we would also retrieve all references corresponding to [INFORM(WELCOME); REQUEST(NAME)] as they share the INFORM(WELCOME) attribute.

In the LOLS framework, we also obtain the training signal via $\pi$. In this work, this is similar to how we calculate $\pi^{r e f}$ but instead of greedily generating the rest of the sentence for each $x_{t+1}^{i}$, we generate by sampling using $\pi$. In order to allow a broader exploration and generate a more consistent signal when sampling, multiple hypotheses are produced and precision is averaged over them.

\section{Experiments}

The following experimental analysis is performed on the MultiWOZ dataset (Budzianowski et al., 2018) which contains human-to-human written conversations, annotated with corresponding MRs. The conversations concern a user trying to use a virtual assistant to perform certain tasks, e.g. book a restaurant or a taxi, find attractions. The dataset is comprised of 55026, 7290 and 7291 utterances for training, validation and testing respectively. In the training set, there are 486 different attributes, 8635 unique MRs and a total of 46671 distinct sentences. We note that scarcity of data is one of the major 
challenges of concept-to-text NLG, and that MultiWOZ is one of the largest and more diverse datasets available. Both DSTC8 and DSTC9 challenges use MultiWOZ in their tasks ${ }^{1}$.

\subsection{Evaluation Metrics}

To measure the diversity of the outputs we compute Self-BLEU (Zhu et al., 2018) and diversity-n (Li et al., 2016). In our experiments, we will be reporting 1 - Self-BLEU to make the score easily interpretable (the higher the score, the more diverse the system output is), while for distinct$\mathrm{n}$ we provide the percentage of distinct $\mathrm{n}$-grams $(n=1,2,4)$ and distinct whole sentences.

Correctness of the output is evaluated with slot error (Wen et al., 2015a, ERR), i.e. the percentage of values in the MR that are missing, repeated or hallucinated in the output. Overall performance is evaluated with BLEU-4, METEOR (Lavie and Agarwal, 2007) and MoverScore (Zhao et al., 2019). We should note that word overlap metrics can be unreliable when evaluating systems with a high level of diversity in the output. Since every MR is aligned with a limited set of references, more diversity will lead to less overlap between the output and the references. BLEU is particularly problematic, as it has been shown not to be a reliable discriminator between high quality systems even when not considering a particularly diverse output (Novikova et al., 2017). For this reason, we further support our experiments with human evaluation.

\subsection{System Configurations}

Apart from the experiments with SpaceFusion (Gao et al., 2019), all our experiments make use of the Semantically Conditioned Long Short-term Memory (SCLSTM) architecture, proposed by (Wen et al., 2015b), as the underlying NLG model. While recent architectures have been adapted to take advantage of large pretrained language models (Peng et al., 2020), we opt not to use them here as related work does not exploit external data either. For our meta-classifier, we initialised using a single iteration of Exact Imitation over the full dataset. Due to time constraints, for the following training iterations with any IL framework, only $10 \%$ randomly selected sentences were used. We evaluated the meta-classifiers generated by our last iteration. For the MMI objective function we implemented

\footnotetext{
${ }^{1} \mathrm{https} / / /$ sites.google.com/dstc.community/dstc8/tracks, https://sites.google.com/dstc.community/dstc9/tracks
}

MMI-antiLM as suggested by Li et al. (2016). At decoding, the MMI-antiLM parameters were set as $\lambda=0.5$ and $g=5$. Beam Search and MMI are performed with beam size $=10$. SpaceFusion was trained using the configuration provided with the code. Tests on different settings did not achieve significant improvements. Values for the random vector $r$ were generated in the range $-5,5$. For First Word Control, we selected all the words that appear more than 60 times as first word in the training references, resulting in a set of 67 different possible first words. At inference time, one sentence is generated per each first word. For Top- $k$ and Nucleus Sampling, since parameters $k$ and $p$ are not tunable, we report results for ranges 2-10 for $k$ and $0.10-0.95$ for $p$.

The aforementioned parameters in related work $(\lambda, g, r, k, p)$, were all tuned based on observations of output and diversity metrics. Precise tuning of such manual parameters remains a challenge as word-overlap metrics are unreliable predictors of actual output quality (see Section 5.1).

\subsection{Reranking}

For each input, we generate 10 possible outputs and rerank them according to two criteria. We prioritize utterances with lowest slot error, and then sort them according to their normalised sentence probability. The final output is sampled uniformly from the top 5 most probable remaining sentences. This is applied on all considered models to minimise the effect of random sampling on the results.

\subsection{Analysis of Previous Diversity Methods}

Please consult Table 2 for automatic evaluation metrics. We can see from the low numbers in the diversity metrics (1 - Self-BLEU and distinct-n) that none of previous diversity methods produce much output diversity in concept-to-text NLG. Below, we provide some brief analysis on the results. Beam Search: similarly to what has been reported in open-domain NLG research (Li et al., 2016), Table 2 shows that Beam Search produces greedy-like outputs with minimal variations.

MMI-AntiLM: using Beam Search with MMI as objective function improved the diversity of the output. However, an analysis of the text revealed that the generated sentences do not differ substantially from the ones obtained with maximum-likelihood, and that the achieved diversity was the result of introducing disfluent words within the first $g$ tokens. 


\begin{tabular}{l|cccccccccc} 
& Greedy & $\begin{array}{c}\text { Beam } \\
b=10\end{array}$ & FWC & $\begin{array}{c}\text { MMI } \\
g, \lambda=5,0.5\end{array}$ & $\begin{array}{c}\text { SF } \\
|r|=5\end{array}$ & $\begin{array}{c}\text { Top- } k \\
k=2\end{array}$ & $\begin{array}{c}\text { Nucleus } \\
p=0.84\end{array}$ & IS-E & IS-D & IS-L \\
& & $b$ & & & & \\
\hline BLEU & 0.654 & $\mathbf{0 . 6 6 3}$ & 0.592 & 0.486 & 0.439 & 0.336 & 0.488 & 0.326 & 0.334 & 0.334 \\
METEOR & $\mathbf{0 . 4 9 6}$ & $\mathbf{0 . 4 9 6}$ & 0.479 & 0.479 & 0.332 & 0.400 & 0.434 & 0.393 & 0.395 & 0.395 \\
Mover & $\mathbf{0 . 8 0 4}$ & 0.799 & 0.721 & 0.649 & 0.642 & 0.675 & 0.710 & 0.646 & 0.645 & 0.649 \\
Slot Error & 4.071 & 1.608 & $\mathbf{0 . 3 0 5}$ & 2.091 & 45.218 & 0.830 & 0.753 & 0.897 & 0.762 & 0.897 \\
\hline 1-SB & 0.014 & 0.017 & 0.018 & 0.044 & 0.008 & 0.093 & 0.101 & $\mathbf{0 . 1 0 4}$ & 0.096 & 0.096 \\
Dist-1 & 0.004 & 0.004 & 0.004 & 0.006 & 0.002 & 0.007 & $\mathbf{0 . 0 0 7}$ & $\mathbf{0 . 0 0 7}$ & $\mathbf{0 . 0 0 7}$ & $\mathbf{0 . 0 0 7}$ \\
Dist-2 & 0.022 & 0.024 & 0.023 & 0.049 & 0.013 & 0.066 & $\mathbf{0 . 0 7 2}$ & 0.064 & 0.061 & 0.061 \\
Dist-4 & 0.079 & 0.087 & 0.095 & 0.156 & 0.045 & 0.399 & 0.342 & $\mathbf{0 . 4 2 9}$ & 0.415 & 0.417 \\
Dist-Sent & 0.307 & 0.482 & 0.491 & 0.487 & 0.266 & 0.869 & 0.919 & $\mathbf{0 . 9 6 1}$ & 0.957 & 0.957
\end{tabular}

Table 2: Automatic evaluation results for different methods on diversity. IS-X refers to Informed Sampling trained with either Exact Imitation (IS-E), DAGGER (IS-D) or LOLS (IS-L).

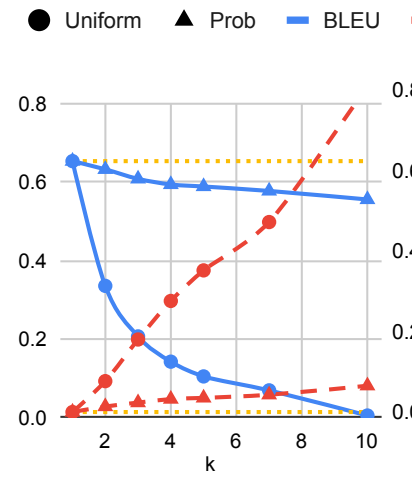

(a) Top- $k$

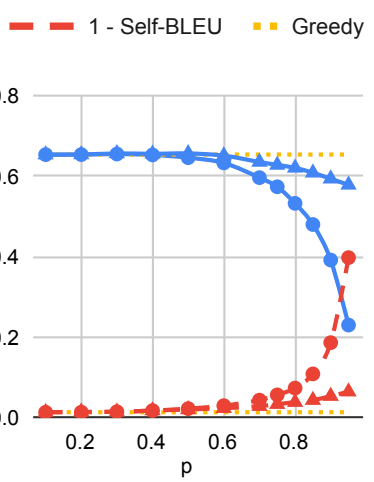

(b) Nucleus Sampling

Figure 3: BLEU and 1 - Self-BLEU for Nucleus Sampling / Top- $k$ with uniform / stochastic sampling.

First Word Control (FWC): despite being proposed for concept-to-text NLG, First Word Control did not achieve more diversity than Beam Search. We have observed that in most cases the forced first word has no major effect on the sentence. For example, for the MR [REQUEST(TAXI-LEAVE)], forcing "Okay", "Alright" or "Great" will not produce diversity as the model will complete the sentence with "What time would you like to leave?".

SpaceFusion (SF): compared to the above methods SpaceFusion obtained the lowest scores for diversity and highest slot error. This makes sense as the method was not designed for concept-totext NLG nor for lexical diversity in general, and the trained autoencoder tends to produce identical or almost identical sentences as the S2S encoder. The joint training collapses the sentence embeddings into a similar representation, preventing the decoder from distinguishing different autoencoder states. This is explained by the strict semantic relation between the MR and the reference, and the similarities within the reference set and the fuse

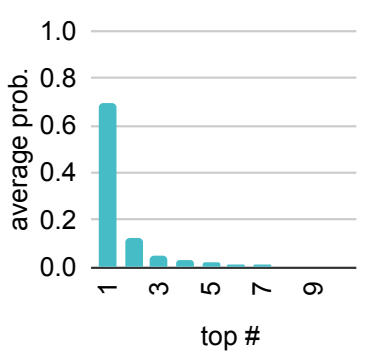

(a) Average prob. distribution

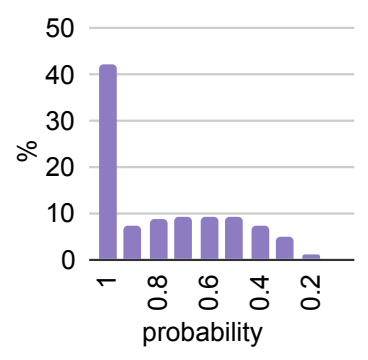

(b) Probability of the top-1
Figure 4: Close up of the distribution produced by the concept-to-text NLG model.

regularisation term. The extremely high slot error can be attributed to the lack of an attention mechanism in SpaceFusion. Widening the range of the random vector added to the latent vectors can increase diversity but not without reducing the relevancy of the sentences further. We conjecture that SpaceFusion might achieve a better performance with an input-optimised model and parameters, but that is beyond the scope of this paper.

Figure 3 show how the quality of the texts produced by Top- $k$ and Nucleus Sampling when paired with stochastic sampling vary as their respective parameters increase. ${ }^{2}$ Despite enlarging the sample pool results in the augmentation of diversity, Top- $k$ and Nucleus Sampling performed comparably across all the parameters, obtaining greedy-like results. Figure 4a shows the average probability of the top-10 words. Since most of the probability mass is clustered in the top 4 words, with the top- 1 taking $70 \%$ of it, we can conclude that stochastic sampling is not appropriate for concept-to-text NLG as little to no diversity would be introduced.

\footnotetext{
${ }^{2}$ We present detailed results in the Appendix.
} 


\subsection{Top- $k$ and Nucleus Sampling Analysis}

In addition to stochastic sampling, Figure 3 shows the performance of Top- $k$ and Nucleus Sampling when paired with uniform sampling. For Top- $k$ (Figure 3a), while the diversity in the text increases drastically, the BLEU score drops exponentially over $k$. The score halves even for $k=2$ (one step beyond greedy decoding) and reaches a 0.005 BLEU score at $k=10$. Figure $4 \mathrm{~b}$ shows that $42 \%$ of the generated words have a probability of 1.0 (or nearly 1.0). Even though diversity methods aim to reduce the bias towards highly probable words, it is safe to assume that in concept-to-text NLG, words with a probability of 1.0 are likely to be the sole correct output. For this reason, when $k$ increases, errors on these cases become more probable. In addition, it is fairly reasonable to trust the low scores of word-overlap metrics on the incorrectness of the output produced by top- $k$ due to their high correlation with human judgements when evaluating low-quality text (Zhao et al., 2019).

On the other hand, Nucleus Sampling paired with uniform sampling is able to introduce diversity in a more controlled way, outperforming Top- $k$ by maintaining a high level of BLEU while steadily increasing the diversity generated. (Figure 3b). We note that Nucleus Sampling can achieve any desired level of diversity through different values of $p$. However, picking an optimal value for $p$ is not straightforward as the effect of each level of diversity to the quality of the output is unreliably measured by the word overlap metrics.

\subsection{Evaluation of Informed Sampling}

Table 2 also shows our three Informed Sampling models trained with Exact Imitation (IS-E), DAGGER (IS-D) and LOLS (IS-L). All the configurations obtained comparable automatic evaluation results, suggesting that the benefits of LOLS do not help in this task. We conjecture this is due to the high quality of the expert policy which provides a reliable and representative training signal for the diversity that the NLG is capable of producing correctly.

Compared to previous methods our approaches show a much higher level of diversity in the output. However, we observe a significant drop in the word overlap metrics (BLEU-4, METEOR and MoverScore). As we mentioned in section 5.1, these metrics rely on a limited set of evaluation references, and are unfortunately unreliable when there is a high level of diversity in the output. We consider

\begin{tabular}{l|cc|cc}
\multirow{2}{*}{} & \multicolumn{2}{|c|}{ Fluency } & \multicolumn{2}{c}{ Adequacy } \\
\cline { 2 - 5 } & raw & z-score & raw & z-score \\
\hline Greedy & $\mathbf{8 2 . 5 5 5}$ & 0.334 & $\mathbf{8 4 . 2 3 3}$ & 0.205 \\
IS-E & 73.892 & 0.028 & 79.790 & 0.057 \\
IS-D & 71.824 & -0.032 & 79.043 & 0.020 \\
IS-L & 73.343 & -0.002 & 79.846 & 0.017 \\
Nucleus & 76.753 & 0.120 & 82.581 & 0.156
\end{tabular}

Table 3: Human Evaluation results.

\begin{tabular}{l|cc|cc}
\multirow{2}{*}{} & \multicolumn{2}{|c|}{ Fluency } & \multicolumn{2}{c}{ Adequacy } \\
\cline { 2 - 5 } & raw & z-score & raw & z-score \\
\hline IS-E & 49.258 & -0.041 & 65.357 & 0.012 \\
IS-D & 53.593 & 0.080 & 64.265 & -0.006 \\
IS-L & $\mathbf{5 4 . 3 2 4}$ & 0.104 & $\mathbf{6 5 . 5 0 9}$ & 0.065 \\
Nucleus & 39.762 & -0.295 & 60.561 & -0.017
\end{tabular}

Table 4: Human Evaluation results for texts always sampling the last word of the reduced sample pool.

Self-BLEU and distinct- $n$ to be accurate as they do not rely on references. To better determine the output's quality, we perform human evaluation.

For human evaluation we include the output of Nucleus Sampling and greedy decoding. To further focus the human evaluation solely on output quality, we aim to keep the level of diversity across systems as close as possible. The behavior of greedy decoding is not adjustable, but we can adjust the level of diversity of Nucleus Sampling using different $p$ values. Unfortunately, we cannot use the development data to pick $p$ as we observed it leads to different Self-BLUE values in the test set which would compromise the comparison. We set $p=0.84$ as that leads to the same Self-BLEU as our systems on the test data. This also leads to a higher BLEU score by 0.16 points, but the difference for semantic similarity based metrics is more marginal, with a difference of only 0.04 for METEOR and 0.06 for MoverScore. We note that the inclusion of $\mathrm{Nu}$ cleus Sampling and greedy decoding is to provide context for the human participants, and not to directly compare against them as methods. Greedy decoding is more fluent as it produces no diversity, and Nucleus Sampling is optimized in an unrealistically favorable manner, as there is no established methodology to tune the parameter $p$ otherwise.

We evaluate the fluency and adequacy of the texts via Direct Assessment (Graham et al., 2017); a human evaluation framework that has been employed on MT (Bojar et al., 2018), surface realisation (Mille et al., 2018) and video captioning 
(Awad et al., 2019) output. We used the publicly available code of Direct Assessment ${ }^{3}$ to setup tasks on the Amazon Mechanical Turk (AMT) platform.

To minimise correlation between the criteria, separate tasks were created asking participants to assess the fluency and adequacy of the provided texts; a 100-point Likert scale was used. For fluency, participants were asked to judge how grammatical and natural the text was. The task for adequacy was more complicated as participants were asked to compare the text with a checklist of snippets that it should include. We generated the checklist of snippets through simple rule based NLG (i.e. templates). Every text was evaluated by at least 3 participants. We limited the crowd-workers that could participate in the tasks to those residing in Englishspeaking countries, and who had a high acceptance rate. Even so, after consulting the participants' reliability based on the Direct Assessment platform analysis, we had to filter out $27 \%$ and $50 \%$ of those who assessed fluency and adequacy respectively.

We sampled 1500 texts from each of the different Informed Sampling configurations, Nucleus Sampling and greedy inference. Table 3 shows the raw and mean standardised z-scores of the human assessments. To determine whether the observed differences were statistically significant we used the Wilcoxon rank sum test. On both fluency and adequacy the greedy model is the best, while IS-E and Nucleus are comparable on fluency. All other configurations have no statistically significant difference between them. This confirms that Informed sampling learns a level of diversity that the NLG model can generate without particularly hurting the output's quality when compared to an unrealistic optimization of Nucleus Sampling. While fluency and adequacy is lower than greedy inference (as is to be expected), the gain in diversity is significant.

\subsection{Sample pool analysis}

To better assess the edge cases of the decoding strategies, we generate 750 texts from Nucleus and each Informed Sampling configuration by always picking the least probable word in the range that each method returns. This will help us determine the quality of the texts for which the NLG model is least confident, but the decoding strategies still consider to be reliable enough to generate. Table 4 shows the raw and mean standardised z-scores for

\footnotetext{
${ }^{3}$ https://github.com/ygraham/crowd-alone
}

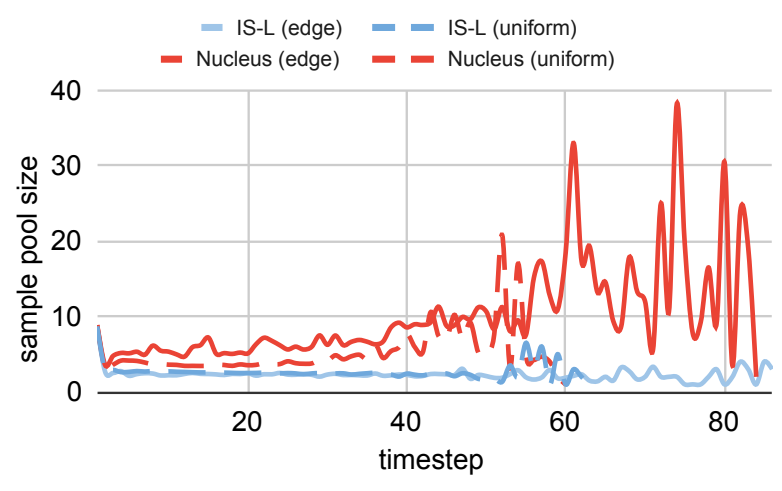

Figure 5: Average sample pool size over decoding.

this setting. ${ }^{4}$ Again, most configurations show no statistically significant difference between them, with the exception of IS-L and Nucleus on fluency. This shows that Informed Sampling is better at determining edge cases where it can reliably generate diverse output without hurting quality.

In addition, Figure 6 shows how the sample pool varies over the course of decoding a sentence (i.e. at each timestep) for each decoding strategy. We compare the behavior of IS-L and Nucleus, when decoding the sentences by either uniform sampling or always picking the least probable (edge) word. ${ }^{5}$ IS-L generally begins with a larger pool size at timestep $t=0$, indicating that it considers more diverse ways to begin the sentences. Overall, we observe that the pool size for Nucleus is larger and becomes even larger and more inconsistent at later timesteps. This is especially prevalent when picking the last word, which suggests that Nucleus leads the underlying NLG model to become less confident, possibly due to error propagation. On the other hand, IS-L demonstrates more consistent behavior, reducing its pool size over time as fewer sentence variations become available.

\section{Conclusion}

In this paper, we proposed Informed Sampling which employs a meta-classifier exploiting diversity-specific training signals to determine which words in the decoding distribution lead to reliably diverse generation. Due to the lack of explicit training signal for diversity, we adapted three Imitation Learning frameworks and showed that their application helps Informed Sampling determine the level of diversity that the underlying NLG

\footnotetext{
${ }^{4}$ Automatic metrics results are included in the appendix.

${ }^{5}$ IS-E and IS-D produce sample pools similar to IS-L. Full plot of Figure 6 is provided in the Appendix.
} 
model is comfortable to produce. Our experimental results show that Informed Sampling leads to highly diverse output while minimising the cost to the quality of the text. We also show that $I n$ formed Sampling is better than previous work at determining the edge cases where it can still reliably generate diverse output even though the NLG model assigns a lower probability. Additionally, we presented a thorough analysis of open-domain diversity methods applied to concept-to-text NLG.

Informed Sampling is agnostic to the underlying model; its input consists of hidden states/embeddings and a probability distribution that can be obtained from almost any language generation model. In future work, we aim to extend Informed Sampling to other language generation tasks, e.g. machine translation and open-domain NLG. Additionally, it would be interesting to explore the application of Informed Sampling over the probability distribution of large pretrained models.

\section{References}

George Awad, Asad Butt, Keith Curtis, Yooyoung Lee, Jonathan Fiscus, Afzal Godil, David Koy, Andrew Delgado, Alan Smeaton, Yvette Graham, Wessel Kraaij, Georges Quénot, João Magalhães, David Semedo, and Saverio Blasi. 2019. Trecvid 2018: Benchmarking video activity detection, video captioning and matching, video storytelling linking and video search. In Proceedings of TRECVID 2018, Gaithersburg, MD, United States.

Ondřej Bojar, Christian Federmann, Mark Fishel, Yvette Graham, Barry Haddow, Philipp Koehn, and Christof Monz. 2018. Findings of the 2018 conference on machine translation (WMT18). In Proceedings of the Third Conference on Machine Translation: Shared Task Papers, pages 272-303, Belgium, Brussels. Association for Computational Linguistics.

Paweł Budzianowski, Tsung-Hsien Wen, Bo-Hsiang Tseng, Iñigo Casanueva, Stefan Ultes, Osman Ramadan, and Milica Gašić. 2018. MultiWOZ - a large-scale multi-domain wizard-of-Oz dataset for task-oriented dialogue modelling. In Proceedings of the 2018 Conference on Empirical Methods in Natural Language Processing, pages 5016-5026, Brussels, Belgium. Association for Computational Linguistics.

Kai-Wei Chang, Akshay Krishnamurthy, Alekh Agarwal, John Langford, and Hal Daumé III. 2015. Learning to search better than your teacher. Proceedings of the 32nd International Conference on Machine Learning, Lille, France, 2015. JMLR: $W \& C P$ volume 37.
Jan Milan Deriu and Mark Cieliebak. 2017. End-toend trainable system for enhancing diversity in natural language generation. In End-to-End Natural Language Generation Challenge (E2E NLG), 2017.

Ondřej Dušek, Jekaterina Novikova, and Verena Rieser. 2018. Findings of the E2E NLG challenge. In Proceedings of the 11th International Conference on Natural Language Generation, pages 322-328, Tilburg University, The Netherlands. Association for Computational Linguistics.

Angela Fan, Mike Lewis, and Yann Dauphin. 2018. Hierarchical neural story generation. In Proceedings of the 56th Annual Meeting of the Association for Computational Linguistics (Volume 1: Long Papers), pages 889-898, Melbourne, Australia. Association for Computational Linguistics.

Xiang Gao, Sungjin Lee, Yizhe Zhang, Chris Brockett, Michel Galley, Jianfeng Gao, and Bill Dolan. 2019. Jointly optimizing diversity and relevance in neural response generation. In NAACL-HLT (1), pages 1229-1238.

Yoav Goldberg and Joakim Nivre. 2013. Training deterministic parsers with non-deterministic oracles. Transactions of the Association for Computational Linguistics, 1:403-414.

James Goodman, Andreas Vlachos, and Jason Naradowsky. 2016. Noise reduction and targeted exploration in imitation learning for abstract meaning representation parsing. In Proceedings of the 54th Annual Meeting of the Association for Computational Linguistics (Volume 1: Long Papers), pages 111, Berlin, Germany. Association for Computational Linguistics.

Yvette Graham, Timothy Baldwin, Alistair Moffat, and Justin Zobel. 2017. Can machine translation systems be evaluated by the crowd alone. Natural Language Engineering, 23(1):3-30.

Ari Holtzman, Jan Buys, Li Du, Maxwell Forbes, and Yejin Choi. 2020. The curious case of neural text degeneration. In International Conference on Learning Representations.

Diederick P Kingma and Jimmy Ba. 2015. Adam: A method for stochastic optimization. In 3rd International Conference on Learning Representations, ICLR 2015, San Diego, CA, USA, May 7-9, 2015, Conference Track Proceedings.

Gerasimos Lampouras and Andreas Vlachos. 2016. Imitation learning for language generation from unaligned data. In Proceedings of COLING 2016, the 26th International Conference on Computational Linguistics: Technical Papers, pages 1101-1112, Osaka, Japan. The COLING 2016 Organizing Committee.

Alon Lavie and Abhaya Agarwal. 2007. METEOR: An automatic metric for MT evaluation with high levels 
of correlation with human judgments. In Proceedings of the Second Workshop on Statistical Machine Translation, pages 228-231, Prague, Czech Republic. Association for Computational Linguistics.

Jiwei Li, Michel Galley, Chris Brockett, Jianfeng Gao, and Bill Dolan. 2016. A diversity-promoting objective function for neural conversation models. In Proceedings of the 2016 Conference of the North American Chapter of the Association for Computational Linguistics: Human Language Technologies, pages 110-119, San Diego, California. Association for Computational Linguistics.

Hongyuan Mei, Mohit Bansal, and Matthew R. Walter. 2016. What to talk about and how? selective generation using LSTMs with coarse-to-fine alignment. In Proceedings of the 2016 Conference of the North American Chapter of the Association for Computational Linguistics: Human Language Technologies, pages 720-730, San Diego, California. Association for Computational Linguistics.

Simon Mille, Anja Belz, Bernd Bohnet, Yvette Graham, Emily Pitler, and Leo Wanner. 2018. The first multilingual surface realisation shared task (SR'18): Overview and evaluation results. In Proceedings of the First Workshop on Multilingual Surface Realisation, pages 1-12, Melbourne, Australia. Association for Computational Linguistics.

Jekaterina Novikova, Ondřej Dušek, Amanda Cercas Curry, and Verena Rieser. 2017. Why we need new evaluation metrics for NLG. In Proceedings of the 2017 Conference on Empirical Methods in Natural Language Processing, pages 2241-2252, Copenhagen, Denmark. Association for Computational Linguistics.

Kishore Papineni, Salim Roukos, Todd Ward, and WeiJing Zhu. 2002. BLEU: a method for automatic evaluation of machine translation. In Proceedings of the 40th annual meeting on association for computational linguistics, pages 311-318. Association for Computational Linguistics.

Baolin Peng, Chenguang Zhu, Chunyuan Li, Xiujun $\mathrm{Li}$, Jinchao Li, Michael Zeng, and Jianfeng Gao. 2020. Few-shot natural language generation for task-oriented dialog. Empirical Methods in Natural Language Processing (EMNLP).

Stéphane Ross, Geoffrey Gordon, and Drew Bagnell. 2011. A reduction of imitation learning and structured prediction to no-regret online learning. In Proceedings of the fourteenth international conference on artificial intelligence and statistics, pages 627 635 .

Andreas Vlachos and Stephen Clark. 2014. A new corpus and imitation learning framework for contextdependent semantic parsing. Transactions of the Association for Computational Linguistics, 2:547-560.
Tsung-Hsien Wen, Milica Gašić, Dongho Kim, Nikola Mrkšić, Pei-Hao Su, David Vandyke, and Steve Young. 2015a. Stochastic language generation in dialogue using recurrent neural networks with convolutional sentence reranking. In Proceedings of the 16th Annual Meeting of the Special Interest Group on Discourse and Dialogue, pages 275-284, Prague, Czech Republic. Association for Computational Linguistics.

Tsung-Hsien Wen, Milica Gasic, Nikola Mrkšić, PeiHao Su, David Vandyke, and Steve Young. 2015b. Semantically conditioned lstm-based natural language generation for spoken dialogue systems. In Proceedings of the 2015 Conference on Empirical Methods in Natural Language Processing, pages 1711-1721, Lisbon, Portugal. Association for Computational Linguistics.

Yizhe Zhang, Michel Galley, Jianfeng Gao, Zhe Gan, Xiujun Li, Chris Brockett, and Bill Dolan. 2018 Generating informative and diverse conversational responses via adversarial information maximization. In Advances in Neural Information Processing Systems 31, pages 1810-1820.

Tiancheng Zhao, Ran Zhao, and Maxine Eskenazi. 2017. Learning discourse-level diversity for neural dialog models using conditional variational autoencoders. In Proceedings of the 55th Annual Meeting of the Association for Computational Linguistics (Volume 1: Long Papers), pages 654-664, Vancouver, Canada. Association for Computational Linguistics.

Wei Zhao, Maxime Peyrard, Fei Liu, Yang Gao, Christian M. Meyer, and Steffen Eger. 2019. MoverScore: Text generation evaluating with contextualized embeddings and earth mover distance. In Proceedings of the 2019 Conference on Empirical Methods in Natural Language Processing and the 9th International Joint Conference on Natural Language Processing (EMNLP-IJCNLP), pages 563-578, Hong Kong, China. Association for Computational Linguistics.

Yaoming Zhu, Sidi Lu, Lei Zheng, Jiaxian Guo, Weinan Zhang, Jun Wang, and Yong Yu. 2018. Texygen: A benchmarking platform for text generation models. In The 41st International ACM SIGIR Conference on Research \& Development in Information Retrieval, SIGIR '18, pages 1097-1100. ACM. 


\section{A System Configurations}

This section is similar to the system configurations section of the main paper, but includes many more configuration details.

Apart from the experiments with SpaceFusion (Gao et al., 2019), all our experiments make use of SCLSTM as underlying NLG model. Using the implementation provided by Budzianowski et al. $(2018)^{6}$, the model has been trained with 4 hidden layers, states of size 100 and 0.25 dropout, Adam (Kingma and $\mathrm{Ba}, 2015$ ) as loss optimiser, learning rate of 0.005 and gradient clipping at 0.5 . Early stopping was applied when validation loss did not decrease within 6 epochs.

For our meta-classifier, we used 3 linear/ReLU layers with 512 as hidden state size, trained for 30 epochs per dataset iteration with Stochastic Gradient Descent and a learning rate of 0.05. For LOLS, exponential decay is performed with a parameter $\beta$ of 0.1 . We initialised using a single iteration of Exact Imitation over the full dataset. Due to time constraints, for the following training iterations with any IL framework, only $10 \%$ randomly selected sentences were used. We evaluated the meta-classifiers generated by our last iteration.

In addition to our proposed method, we explored six different techniques for diversity: sampling Beam Search, First Word Control (Deriu and Cieliebak, 2017), MMI-AntiLM (Li et al., 2016), SpaceFusion (Gao et al., 2019), Top- $k$ (Fan et al., 2018) and Nucleus Sampling (Holtzman et al., 2020). Beam Search and MMI are performed with beam size $=10$ and the sentences are selected among the top 10 beams with the criteria described in Section 5.3. For the MMI objective function we implemented MMI-antiLM as suggested by Li et al. (2016). For the auxiliary language model, we used a 2-layer, 650 vector size LSTM, trained for 40 epochs on the MultiWOZ references. At decoding, the MMI-antiLM parameters were set as $\lambda=0.5$ and $g=5$. SpaceFusion was trained using the configuration provided with the code. Tests on different settings did not achieve significant improvements. Values for the random vector $r$ were generated in the range $-5,5$. For First Word Control, we selected all the words that appear more than 60 times as first word in the training references, resulting in a set of 67 different possible first words. SCLSTM was modified as in Deriu and Cieliebak (2017) and trained with the configuration

\footnotetext{
${ }^{6}$ https://github.com/andy 194673/nlg-sclstm-multiwoz/
}

described above. At inference time, one sentence is generated per each first word and the output is selected with the criteria described in Section 5.3. For Top- $k$ and Nucleus Sampling, since parameters $k$ and $p$ are not tunable, we report results for ranges 2-10 for $k$ and 0.10-0.95 for $p$.

The aforementioned parameters in related work $(\lambda, g, r, k, p)$, were all tuned based on observations of output and diversity metrics.

\section{B Complete Results}

Tables 5 and 6 show detailed results of automatic metrics for Top- $k$ across different values of $k$ for uniform and stochastic sampling respectively. Similarly, Tables 7 and 8 show detailed results for $\mathrm{Nu}-$ cleus Sampling across different values of $p$. These results are corresponding to those shown in Figure 3 of the main paper.

Table 9 shows the results for Space Fusion by varying the range of the $r$ random vector added to the input latent variable. Similarly to $k$ and $p$, performance and diversity are inversely correlated when the range is widened. However, this change does not considerably affect the slot error, which remains drastically higher than other systems.

Table 10 shows automatic results for the edge case experiment presented in Section 5.6 of the main paper, and correspond to the human evaluation experiments summarised in Table 4. Similarly to the results presented in Table 2, Nucleus Sampling achieved the highest BLEU score. However, all systems performed similarly according to METEOR and MoverScore, while Informed Sampling methods produced outputs with fewer slot errors than Nucleus. Diversity metrics are not included as diversity comparison is not informative when performed on experiments where the word choice at each timestep is forced (here to the least probable word) rather than sampled.

\section{Examples}

Table 11 and 12 show some output examples produced by each diversity method after reranking based on slot error and normalised sentence probability. We present the top 3 sentences, and do not filter out repeated sequences (as in our evaluation).

In the first example, for the meaning representation [INFORM(TRAIN-REF $=\mathrm{ABC} 123$ ), INFORM(TRAIN-PRICE = 10)], all the systems generated sentences with structures similar to the greedily-decoded output. Beam Search, MMI and 


\begin{tabular}{l|ccccccc} 
& $\mathrm{k}=1$ & $\mathrm{k}=2$ & $\mathrm{k}=3$ & $\mathrm{k}=4$ & $\mathrm{k}=5$ & $\mathrm{k}=7$ & $\mathrm{k}=10$ \\
\hline BLEU & 0.654 & 0.336 & 0.207 & 0.144 & 0.105 & 0.069 & 0.005 \\
METEOR & 0.496 & 0.400 & 0.368 & 0.352 & 0.343 & 0.329 & 0.253 \\
Mover & 0.804 & 0.675 & 0.604 & 0.559 & 0.533 & 0.497 & 0.368 \\
Slot Error & 4.071 & 0.830 & 1.329 & 2.192 & 2.819 & 4.461 & 30.667 \\
\hline 1 - SB & 0.014 & 0.093 & 0.199 & 0.297 & 0.375 & 0.483 & 0.846 \\
Dist-1 & 0.004 & 0.007 & 0.008 & 0.008 & 0.008 & 0.008 & 0.007 \\
Dist-2 & 0.022 & 0.066 & 0.096 & 0.117 & 0.134 & 0.162 & 0.400 \\
Dist-4 & 0.079 & 0.399 & 0.638 & 0.771 & 0.844 & 0.919 & 0.999 \\
Dist-Sent & 0.307 & 0.869 & 0.943 & 0.978 & 0.985 & 0.990 & 0.996
\end{tabular}

Table 5: Complete results for Top- $k$ with uniform sampling.

\begin{tabular}{l|ccccccc} 
& $\mathrm{k}=1$ & $\mathrm{k}=2$ & $\mathrm{k}=3$ & $\mathrm{k}=4$ & $\mathrm{k}=5$ & $\mathrm{k}=7$ & $\mathrm{k}=10$ \\
\hline BLEU & 0.654 & 0.633 & 0.609 & 0.594 & 0.589 & 0.578 & 0.556 \\
METEOR & 0.496 & 0.489 & 0.480 & 0.476 & 0.473 & 0.470 & 0.461 \\
Mover & 0.804 & 0.797 & 0.784 & 0.780 & 0.775 & 0.768 & 0.752 \\
Slot Error & 4.071 & 0.728 & 0.652 & 0.643 & 0.482 & 0.576 & 0.559 \\
\hline 1 - SB & 0.014 & 0.028 & 0.038 & 0.047 & 0.050 & 0.058 & 0.081 \\
Dist-1 & 0.004 & 0.005 & 0.005 & 0.006 & 0.006 & 0.006 & 0.007 \\
Dist-2 & 0.022 & 0.032 & 0.039 & 0.044 & 0.046 & 0.051 & 0.063 \\
Dist-4 & 0.079 & 0.138 & 0.177 & 0.204 & 0.216 & 0.238 & 0.289 \\
Dist-Sent & 0.307 & 0.592 & 0.673 & 0.708 & 0.738 & 0.770 & 0.819
\end{tabular}

Table 6: Complete results for Top- $k$ with stochastic sampling.

First Word Control behaved as described in Section 5.4. The rest of the systems were able to introduce some degree of diversity, while Nucleus Sampling and Space Fusion produced repeated sentences.

On the other hand, for the [INFORM(TRAIN-REF $=\mathrm{ABC} 123)$, INFORM $($ TRAIN-PRICE $=10)]$, all the systems with the exception of Beam Search and MMI, produced diverse sentences. However, First Word Control, and Space Fusion generated some irrelevant content, while Top- $k$, Nucleus Sampling, and IS-E present some disfluency.

Table 13 illustrates some output examples generated by Greedy (as benchmark), Nucleus and Informed Sampling for the edge case experiment presented in Section 5.6. These examples correspond to the human evaluation experiments summarised in Table 4 and Table 10. Overall, neither Informed Sampling models nor Nucleus sampling were able to generate consistently correct and fluent outputs. However, the table illustrates some examples of how catastrophic error propagation can be when non-reliable words are sampled. Specifically, for the first MR, Nucleus Sampling produced a nonsensical sentence which we attribute mainly at the generation of the tokens "british" and ",". Informed Sampling models also suffer from error propaga- tion (as seen on the second MR), but its effects are not as frequent or severe as when using Nucleus Sampling.

\section{Human evaluation platform examples}

Figures 7 and 8 show examples of the evaluation platform as shown to the human participants of Amazon Mechanical Turn. Figure 7 asks the participants to rate the fluency of the text, while Figure 8 is used to rate adequacy. For the latter, people were asked to compare the text with a checklist of snippets that it should include. The checklist of snippets was generated through simple rule based NLG (i.e. manually authored templates). 


\begin{tabular}{l|cccccccccccc} 
& $\mathrm{p}=0.1$ & $\mathrm{p}=0.2$ & $\mathrm{p}=0.3$ & $\mathrm{p}=0.4$ & $\mathrm{p}=0.5$ & $\mathrm{p}=0.6$ & $\mathrm{p}=0.7$ & $\mathrm{p}=0.75$ & $\mathrm{p}=0.8$ & $\mathrm{p}=0.85$ & $\mathrm{p}=0.9$ & $\mathrm{p}=0.95$ \\
\hline BLEU & 0.654 & 0.654 & 0.655 & 0.653 & 0.646 & 0.633 & 0.596 & 0.574 & 0.535 & 0.480 & 0.392 & 0.230 \\
METEOR & 0.495 & 0.495 & 0.497 & 0.496 & 0.496 & 0.497 & 0.470 & 0.461 & 0.449 & 0.432 & 0.401 & 0.360 \\
Mover & 0.804 & 0.804 & 0.804 & 0.804 & 0.799 & 0.789 & 0.766 & 0.751 & 0.7333 & 0.704 & 0.656 & 0.557 \\
Slot Error & 4.080 & 3.674 & 2.878 & 2.167 & 1.261 & 0.906 & 0.770 & 0.643 & 0.719 & 0.982 & 1.244 & 3.801 \\
\hline 1 - SB & 0.014 & 0.014 & 0.015 & 0.018 & 0.023 & 0.030 & 0.044 & 0.057 & 0.074 & 0.109 & 0.187 & 0.398 \\
Dist-1 & 0.004 & 0.004 & 0.004 & 0.004 & 0.005 & 0.005 & 0.006 & 0.006 & 0.007 & 0.008 & 0.009 & 0.009 \\
Dist-2 & 0.022 & 0.022 & 0.023 & 0.025 & 0.030 & 0.034 & 0.042 & 0.049 & 0.058 & 0.075 & 0.113 & 0.215 \\
Dist-4 & 0.079 & 0.080 & 0.082 & 0.092 & 0.113 & 0.141 & 0.190 & 0.229 & 0.280 & 0.359 & 0.502 & 0.760 \\
Dist-Sent & 0.307 & 0.310 & 0.331 & 0.389 & 0.480 & 0.588 & 0.727 & 0.799 & 0.863 & 0.924 & 0.957 & 0.956
\end{tabular}

Table 7: Complete results for Nucleus Sampling with uniform sampling.

\begin{tabular}{l|cccccccccccc} 
& $\mathrm{p}=0.1$ & $\mathrm{p}=0.2$ & $\mathrm{p}=0.3$ & $\mathrm{p}=0.4$ & $\mathrm{p}=0.5$ & $\mathrm{p}=0.6$ & $\mathrm{p}=0.7$ & $\mathrm{p}=0.75$ & $\mathrm{p}=0.8$ & $\mathrm{p}=0.85$ & $\mathrm{p}=0.9$ & $\mathrm{p}=0.95$ \\
\hline BLEU & 0.654 & 0.654 & 0.656 & 0.656 & 0.656 & 0.651 & 0.635 & 0.628 & 0.621 & 0.608 & 0.594 & 0.578 \\
METEOR & 0.495 & 0.495 & 0.497 & 0.498 & 0.497 & 0.496 & 0.489 & 0.486 & 0.483 & 0.478 & 0.473 & 0.468 \\
Mover & 0.804 & 0.804 & 0.805 & 0.805 & 0.806 & 0.804 & 0.794 & 0.786 & 0.790 & 0.779 & 0.772 & 0.763 \\
Slot Error & 4.088 & 3.665 & 2.920 & 2.108 & 1.405 & 0.990 & 0.813 & 0.686 & 0.686 & 0.567 & 0.626 & 0.550 \\
\hline 1 - SB & 0.014 & 0.015 & 0.015 & 0.018 & 0.021 & 0.024 & 0.029 & 0.034 & 0.039 & 0.044 & 0.054 & 0.065 \\
Dist-1 & 0.004 & 0.004 & 0.004 & 0.004 & 0.004 & 0.005 & 0.005 & 0.006 & 0.006 & 0.006 & 0.006 & 0.007 \\
Dist-2 & 0.022 & 0.023 & 0.022 & 0.024 & 0.027 & 0.030 & 0.033 & 0.036 & 0.039 & 0.042 & 0.048 & 0.054 \\
Dist-4 & 0.079 & 0.080 & 0.081 & 0.089 & 0.100 & 0.118 & 0.139 & 0.157 & 0.170 & 0.190 & 0.216 & 0.245 \\
Dist-Sent & 0.307 & 0.309 & 0.327 & 0.374 & 0.448 & 0.528 & 0.603 & 0.646 & 0.678 & 0.718 & 0.750 & 0.789
\end{tabular}

Table 8: Complete results for Nucleus Sampling with stochastic sampling.

\begin{tabular}{l|cccc} 
& $|r|=1.5$ & $|r|=5$ & $|r|=10$ & $|r|=20$ \\
\hline BLEU & 0.466 & 0.439 & 0.341 & 0.233 \\
METEOR & 0.365 & 0.332 & 0.244 & 0.141 \\
Mover & 0.671 & 0.642 & 0.537 & 0.384 \\
Slot Error & 52.98 & 45.218 & 60.344 & 83.299 \\
\hline 1 - SB & 0.002 & 0.008 & 0.025 & 0.045 \\
Dist-1 & 0.002 & 0.003 & 0.003 & 0.004 \\
Dist-2 & 0.007 & 0.013 & 0.020 & 0.026 \\
Dist-4 & 0.019 & 0.045 & 0.099 & 0.142 \\
Dist-Sent & 0.100 & 0.266 & 0.526 & 0.659
\end{tabular}

Table 9: Results for Space Fusion across different hypersphere radius around the latent vectors.

\begin{tabular}{l|cccc} 
& Nucleus & IS-E & IS-D & IS-L \\
& $p=0.84$ & & & \\
\hline BLEU & $\mathbf{0 . 2 4 3}$ & 0.194 & 0.212 & 0.177 \\
METEOR & 0.340 & $\mathbf{0 . 3 5 0}$ & 0.346 & 0.342 \\
Mover & 0.523 & $\mathbf{0 . 5 6 7}$ & 0.560 & 0.563 \\
Slot Error & 24.581 & $\mathbf{1 9 . 3 2 5}$ & 19.350 & 20.112
\end{tabular}

Table 10: Automatic evaluation results for texts always sampling the last word of the reduced sample pool. 


\begin{tabular}{|c|c|}
\hline MR & [INFORM(TRAIN-REF = ABC123), INFORM(TRAIN-PRICE $=10)]$ \\
\hline Greedy & booking was successful, the total fee is $10 \mathrm{GBP}$ payable at the station . reference number is : $\mathrm{ABC} 123$. \\
\hline \multirow{3}{*}{$\begin{array}{l}\text { Beam } \\
\text { Search }\end{array}$} & booking was successful, the total fee is $10 \mathrm{GBP}$ payable at the station . reference number is : ABC123. \\
\hline & booking was successful, the total fee is $10 \mathrm{GBP}$ payable at the station . your reference number is $\mathrm{ABC} 123$. \\
\hline & booking was successful, the total fee is 10 GBP payable at the station . reference number is : ABC123 \\
\hline \multirow{3}{*}{ MMI } & booked has 10 GBP thing . reference number is : $\mathrm{ABC} 123$. \\
\hline & booked has mention fee of $10 \mathrm{GBP}$. reference number is : $\mathrm{ABC} 123$. \\
\hline & booked has mention total fee is $10 \mathrm{GBP}$ payable at the station . reference number is : $\mathrm{ABC} 123$. \\
\hline \multirow{3}{*}{$\begin{array}{l}\text { First } \\
\text { Word } \\
\text { Control }\end{array}$} & booking was successful, the total fee is $10 \mathrm{GBP}$ payable at the station . reference number is : ABC123. \\
\hline & ok. the booking was successful, the total fee is $10 \mathrm{GBP}$ payable at the station . reference number is : $\mathrm{ABC} 123$. \\
\hline & your booking was successful, the total fee is 10 GBP payable at the station . reference number is : $\mathrm{ABC} 123$. \\
\hline \multirow{3}{*}{$\begin{array}{l}\text { Space } \\
\text { Fusion } \\
|r|=5\end{array}$} & booking was successful, the total fee is $10 \mathrm{GBP}$ payable at the station . reference number is : $\mathrm{ABC} 123$. \\
\hline & booking was successful, the total fee is $10 \mathrm{GBP}$ payable at the station . reference number is : ABC123. \\
\hline & the booking was successful, the total fee is $10 \mathrm{GBP}$ payable at the station . reference number is : $\mathrm{ABC} 123$. \\
\hline \multirow{3}{*}{$\begin{array}{l}\text { Top- } k \\
k=2\end{array}$} & booking is complete your reference number is $\mathrm{ABC} 123$ and it will be $10 \mathrm{GBP}$. \\
\hline & your train has booked. your total fee is $10 \mathrm{GBP}$ and your reference number is $\mathrm{ABC} 123$ \\
\hline & booking was completed . the reference is $\mathrm{ABC} 123$ and it will cost $10 \mathrm{GBP}$. \\
\hline \multirow{3}{*}{$\begin{array}{l}\text { Nucleus } \\
\text { Sampling } \\
p=0.84\end{array}$} & booking was successful, the total fee is $10 \mathrm{GBP}$ payable at the station . reference number is : ABC123. \\
\hline & booking was successful, the total fee is $10 \mathrm{GBP}$ payable at the station . reference number is : ABC123. \\
\hline & the total is $10 \mathrm{GBP}$ and your reference number is $\mathrm{ABC} 123$. \\
\hline \multirow{3}{*}{ IS-E } & yes, the booking was successful . your reference number is : $\mathrm{ABC} 123$, the cost is $10 \mathrm{GBP}$. \\
\hline & yes, the booking was successful, you reference number is $\mathrm{ABC} 123.10 \mathrm{GBP}$ payable at the station \\
\hline & your tickets have been reserved . your total is $10 \mathrm{GBP}$, which you can pay at the station, your reference \# is $\mathrm{ABC} 123$ \\
\hline \multirow{3}{*}{ IS-D } & booking was successful. reference number is $10 \mathrm{GBP}$ payable at the station . your reference number is : $\mathrm{ABC} 123$. \\
\hline & your ticket has been booked ! your reference number is : $\mathrm{ABC} 123$, the price is $10 \mathrm{GBP}$. \\
\hline & yes, your tickets have been booked! the cost is $\mathrm{ABC} 123$ and the total cost is $10 \mathrm{GBP}$. \\
\hline \multirow{3}{*}{ IS-L } & great ! booking was successful and the fee is $10 \mathrm{GBP}$ which you can pay at the station . your reference number is: $\mathrm{ABC} 123$. \\
\hline & yes , booking was successful and your total is $10 \mathrm{GBP}$. you can pay that at the station . reference number is $\mathrm{ABC} 123$ \\
\hline & great ! booking was successful! your reference is $\mathrm{ABC} 123$ and you will pay $10 \mathrm{GBP}$ at the station. \\
\hline
\end{tabular}

Table 11: Top 3 outputs for the MR [INFORM(TRAIN-REF = ABC123), INFORM(TRAIN-PRICE = 10)]. RED and BLUE text mark adequacy and fluency errors respectively.

\begin{tabular}{|c|c|}
\hline MR & [INFORM $($ REST-NAME $=$ TANDORI $)$, INFORM $($ REST-AREA $=$ EAST $)]$ \\
\hline Greedy & Tandoori is in the East side . would you like me to book it for you? \\
\hline \multirow{3}{*}{$\begin{array}{l}\text { Beam } \\
\text { Search }\end{array}$} & Tandoori is located in the East side. would you like me to book it for you? \\
\hline & Tandoori is in the East side . would you like me to book it for you? \\
\hline & Tandoori is located in the East side. would you like to book a table? \\
\hline \multirow{3}{*}{ MMI } & Tandoori going is located in the East side \\
\hline & Tandoori going is located in the East side . \\
\hline & Tandoori going is located in the East side . would you like a reservation ? \\
\hline \multirow{3}{*}{$\begin{array}{l}\text { First } \\
\text { Word } \\
\text { Control } \\
\end{array}$} & unfortunately, $\mathrm{i}$ have 4 Indian restaurants . $\mathrm{i}$ have Tandoori in the South and 1 in the West, and 1 in the East . \\
\hline & yes , there are 4 Indian restaurants. Tandoori in in the South and 1 in the West, and 1 in the East . \\
\hline & of course ! there are 4 Indian restaurants in the South, Tandoori in the West and 1 in the East . \\
\hline \multirow{3}{*}{$\begin{array}{l}\text { Space } \\
\text { Fusion } \\
|r|=5\end{array}$} & Tandori is in East. \\
\hline & $\begin{array}{l}\text { i can book you a table for you . i can book you a table for you . how many people and for how many nights and } \\
\text { how many nights and how many people will be staying? }\end{array}$ \\
\hline & i have the Tandori in the East. \\
\hline \multirow{3}{*}{$\begin{array}{l}\text { Top- } k \\
k=2\end{array}$} & i have the Tandoori in East side, shall i book you? \\
\hline & i have a listing in Tandoori , it is in East side, can i make a booking ? \\
\hline & i have a restaurant located at Tandoori in East side , can i make you a reservation at the address of them? \\
\hline \multirow{3}{*}{$\begin{array}{l}\text { Nucleus } \\
\text { Sampling } \\
p=0.84\end{array}$} & Tandoori is in the East side part of town . would you like a reservation? \\
\hline & it 's located in the East side. i can book a reservation at Tandoori if you are interested. \\
\hline & would you like to book a table at Tandoori at the East side , \\
\hline \multirow{3}{*}{ IS-E } & there is a restaurant in the East side that meets those criteria . would you like to try Tandoori? \\
\hline & Tandoori is in the East side part of the city . would you like me to check availability? \\
\hline & $\mathrm{i}$ have a listing in the East side . would you like to book you a reservation at the Tandoori ? \\
\hline \multirow{3}{*}{ IS-D } & Tandoori is a nice restaurant in the East side \\
\hline & i can book Tandoori in the East side, would you like reservations? \\
\hline & Tandoori is in the East side area, shall i book your table? \\
\hline \multirow{3}{*}{ IS-L } & i have a listing for the restaurant in the East side. would you like me to make a reservation at Tandoori for you ? \\
\hline & Tandoori is in the East side side of the city . would you like the reservation ? \\
\hline & Tandoori is an excellent restaurant in the East side. shall i make the reservation ? \\
\hline
\end{tabular}

Table 12: Top 3 outputs for the MR [INFORM(REST-NAME = TANDORI $)$ INFORM(REST-AREA = EAST)]. RED and BLUE text mark adequacy and fluency errors respectively. 


\begin{tabular}{|l|l|}
\hline MR & [INFORM(RESTAURANT-PRICE = EXPENSIVE)] \\
\hline Greedy & it is in the expensive price range . \\
\hline Nucleus & $\begin{array}{l}\text { great the british chinese expensive options, does n't matter when you have it there and just for additional } \\
\text { info and welcome again if you 're interested in books . }\end{array}$ \\
\hline IS-E & sure . they 're in a expensive price . \\
\hline IS-D & its in a expensive price . \\
\hline IS-L & the restaurant is expensive . \\
\hline MR & [INFORM(ATTRACTION-NAME = JELLO GALLERY; KETTLES YARD)] \\
\hline Greedy & cafe jello gallery and kettles yard are both great options . \\
\hline Nucleus & well and the cafe jello gallery on my favorites i were listed are looking for you either of the . \\
\hline IS-E & sure ! i can suggest the cafe jello gallery, kettles yard . \\
\hline IS-D & sure, i would like to suggest cafe jello gallery . \\
\hline IS-L & there 's cafe jello gallery or the kettles yard, or slot-attraction-recommend-name . \\
\hline MR & [REQUEST(BOOKING-DAY; BOOKING-PEOPLE; BOOKING-STAY] \\
\hline Greedy & $\begin{array}{l}\text { i can help you with that . how many people will be staying, and what day will you be arriving, and } \\
\text { how many nights will you be staying ? }\end{array}$ \\
\hline Nucleus & and just what day and time how many people is it staying for you ? \\
\hline IS-E & okay, can you give me the details ? \\
\hline IS-D & okay, can you give me the details ? \\
\hline IS-L & $\begin{array}{l}\text { how long is your stay ? what day do you plan on arriving, for how long are you staying and what time } \\
\text { would like your reservation to book the restaurant for you? }\end{array}$ \\
\hline
\end{tabular}

Table 13: Output text generated by Nucleus, Informed Sampling and Greedy on sample pool edge cases.

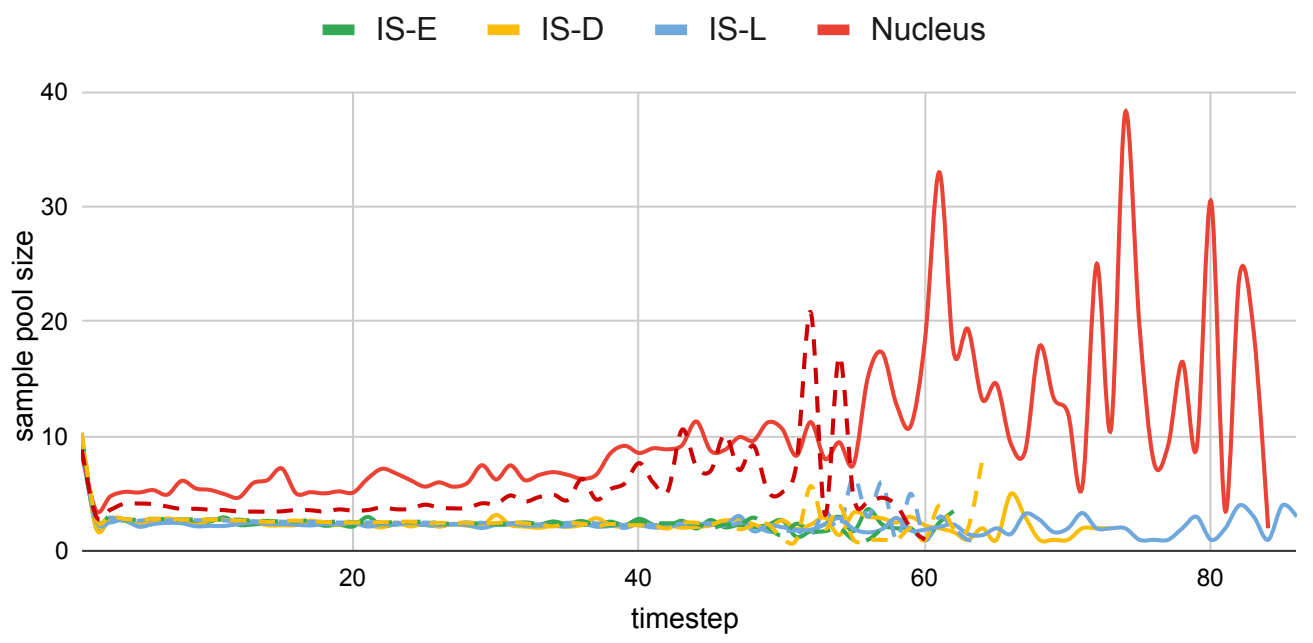

Figure 6: Average sample pool size over decoding. Solid lines correspond to uniform sampling, and dashed lines correspond to sampling the least probable word in the sample (i.e. the edge case experiment).

Read the text below and rate it by how much you agree that:

The text is fluent, i.e. it is grammatically correct and sounds natural.

I have one listing for The Cambridge Belfry that meets your specifications. Shall i book this restaurant?

Figure 7: Evaluation platform for assessment of output fluency. 
Read the text below and rate it by how much you agree that:

The black text adequately expresses the points described in the gray checklist. The black text contains no additional (and irrelevant) information!

-The text should ask the user in what area they are looking for an accommodation.

-The text should mention that it has found about 10 accommodation(s)

I can help you with that. There are about 10 hotels, do you know what part of the city you want to be in?

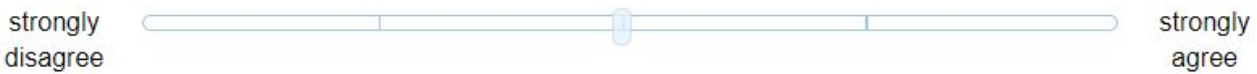

Figure 8: Evaluation platform for assessment of output adequacy. 